\title{
Interpreting the copolymerization of styrene with maleic anhydride and with methyl methacrylate in terms of the bootstrap model
}

Citation for published version (APA):

Klumperman, B., \& O'Driscoll, K. F. (1993). Interpreting the copolymerization of styrene with maleic anhydride and with methyl methacrylate in terms of the bootstrap model. Polymer, 34(5), 1032-1037. https://doi.org/10.1016/0032-3861(93)90226-Z

DOI:

10.1016/0032-3861(93)90226-Z

Document status and date:

Published: 01/01/1993

Document Version:

Publisher's PDF, also known as Version of Record (includes final page, issue and volume numbers)

\section{Please check the document version of this publication:}

- A submitted manuscript is the version of the article upon submission and before peer-review. There can be important differences between the submitted version and the official published version of record. People interested in the research are advised to contact the author for the final version of the publication, or visit the $\mathrm{DOI}$ to the publisher's website.

- The final author version and the galley proof are versions of the publication after peer review.

- The final published version features the final layout of the paper including the volume, issue and page numbers.

Link to publication

\footnotetext{
General rights

- You may freely distribute the URL identifying the publication in the public portal. follow below link for the End User Agreement:

www.tue.nl/taverne

Take down policy

If you believe that this document breaches copyright please contact us at:

openaccess@tue.nl

providing details and we will investigate your claim.
}

Copyright and moral rights for the publications made accessible in the public portal are retained by the authors and/or other copyright owners and it is a condition of accessing publications that users recognise and abide by the legal requirements associated with these rights.

- Users may download and print one copy of any publication from the public portal for the purpose of private study or research.

- You may not further distribute the material or use it for any profit-making activity or commercial gain

If the publication is distributed under the terms of Article 25fa of the Dutch Copyright Act, indicated by the "Taverne" license above, please 


\title{
Interpreting the copolymerization of styrene with maleic anhydride and with methyl methacrylate in terms of the bootstrap model
}

\author{
Bert Klumperman* \\ DSM Research, PO Box 18,6160 MD Geleen, The Netherlands \\ and Kenneth F. O'Driscoll \\ Department of Chemical Engineering, University of Waterloo, Waterloo, Ontario \\ N2L 3G1, Canada \\ (Received 14 January 1992; revised 18 May 1992)
}

\begin{abstract}
The bootstrap model was proposed by Harwood to explain copolymer sequence distribution which is dependent only on copolymer composition in solvents which cause changes in the apparent reactivity ratios for a number of pairs of monomers. This work presents an explicit formulation of the equations describing copolymer composition and sequence distribution in terms of the bootstrap model for both the simple Mayo-Lewis model and the penultimate unit model. Inspection of these equations reveals the necessary and sufficient conditions for sequence distribution to be a function only of copolymer composition. The results of copolymerizations of styrene with methyl methacrylate and with maleic anhydride under various conditions are interpreted in terms of the bootstrap model.
\end{abstract}

(Keywords: copolymerization; bootstrap model; Mayo-Lewis model; penultimate unit model; apparent reactivity ratios; distribution coefficient)

\section{INTRODUCTION}

Harwood ${ }^{1}$ has presented a bootstrap model which deals with solvent effects in free radical copolymerization. In the original paper by Harwood four comonomer pairs show a pronounced solvent effect on determined reactivity ratios when copolymer composition versus monomer feed is studied. All four comonomer pairs consisted of a polar and a non-polar monomer. However, when the copolymer microstructure versus copolymer composition is examined, no such solvent effect is found. A copolymer having the same composition appears to have the same microstructure irrespective of the solvent employed during polymerization. Harwood states that, if the above is the case, the conditional probabilities governing the propagation reaction must be independent of the solvent. The differences observed in the copolymer composition versus monomer feed plots are due to a phenomenon which he calls the bootstrap effect. This effect means that a growing polymer radical influences its own environment. The comonomer ratio available for the growing chain can therefore differ from the global comonomer ratio.

Davis re-analysed the data of San Roman et l. $^{2-4}$, and showed that for a comonomer pair with a relatively small difference in polarity (styrene/methyl

\footnotetext{
* To whom correspondence should be addressed
}

methacrylate) the bootstrap effect can also be observed ${ }^{5}$ The sequence distributions upon which Davis based his examination were measured by n.m.r. spectroscopy ${ }^{6}$. The few publications dealing with the bootstrap model provide only qualitative descriptions of the effect. The aim of this paper is to gain insight into the quantitative aspects of the bootstrap model. Both for the Mayo-Lewis model and for the penultimate unit model the mathematical derivation of the relationship between microstructure and polymer composition will be presented. Furthermore, a method will be proposed for quantification of the distribution coefficient $K$ which was introduced by Harwood in the presentation of his bootstrap model ${ }^{1}$.

\section{THEORY}

For the Mayo-Lewis model the following set of equations is given to describe polymer composition and microstructure as a function of the relevant conditional probabilities $^{7}$ :

$$
\begin{aligned}
\frac{F_{1}}{F_{2}} & =\frac{p_{21}}{p_{12}} \\
F_{111} & =\left(1-p_{12}\right)^{2} \\
F_{112}+F_{211} & =2 p_{12}\left(1-p_{12}\right) \\
F_{212} & =p_{12}^{2}
\end{aligned}
$$


in which: $F_{i}$ is the fraction of monomer $i$ in the copolymer; $F_{i 1 j}$ is the fraction of monomer 1 centred triads with $\Sigma F_{i 1 j}=1$. The conditional probabilities $p_{12}$ and $p_{21}$ are defined as relative propagation rates. For example, $p_{12}$ is the rate of addition of monomer 2 to a chain ending with monomer 1 , where $p_{11}+p_{12}=1$. In terms of the bootstrap model $p_{12}$ and $p_{21}$ can be written as:

$$
\begin{aligned}
& p_{12}=\left(1+r_{1} K q\right)^{-1} \\
& p_{21}=\left(1+\frac{r_{2}}{K q}\right)^{-1}
\end{aligned}
$$

where

$$
q=\frac{\left[M_{1}^{0}\right]}{\left[M_{2}^{0}\right]} \text { and } K=\frac{\left[M_{1}\right] /\left[M_{2}\right]}{\left[M_{1}^{0}\right] /\left[M_{2}^{0}\right]}
$$

and $r_{1}, r_{2}$ have their usual meanings as ratios of propagation rate constants and $\left[M_{i}\right]$ are monomer concentrations. Superscript ${ }^{0}$ refers to global monomer concentration whereas no superscript refers to concentration at the site of propagation.

In any copolymerization in a solvent the reactivity ratios determined are apparent reactivity ratios, $r_{i}^{\mathbf{s}}$, where

$$
\begin{aligned}
& r_{1}^{\mathrm{s}}=r_{1} K^{\mathrm{s}} \\
& r_{2}^{\mathrm{s}}=r_{2} / K^{\mathrm{s}}
\end{aligned}
$$

and $K^{\mathrm{s}}$ is the distribution coefficient as defined in equation ( 7 ) in the solvent.

It should be emphasized that independence of the conditional probabilities upon solvent most probably occurs with independence of the monomer ratio at the site of propagation upon solvent. This means that in theory a 'real' reactivity ratio could be defined related to the monomer ratio at the site of propagation. This monomer ratio cannot be determined easily. It is, however, unnecessary to define real reactivity ratios, since the only objective is to relate the apparent reactivity ratios to a reference state. For the sake of quantification we can arbitrarily define such a reference state. For convenience we define the distribution coefficient to be unity $\left(K^{\mathrm{b}}=1\right)$ in bulk copolymerization, although the global monomer ratio in a bulk copolymerization may also differ from the monomer ratio at the site of propagation.

It follows that when reactivity ratios, $r_{i}^{\mathrm{b}}$, have been determined in bulk:

$$
\begin{aligned}
& \frac{r_{1}^{\mathrm{s}}}{r_{1}^{\mathrm{b}}}=K^{\mathrm{s}} \\
& \frac{r_{2}^{\mathrm{b}}}{r_{2}^{\mathrm{s}}}=K^{\mathrm{s}}
\end{aligned}
$$

and that independent of the solvent employed:

$$
r_{1}^{\mathrm{s}} r_{2}^{\mathrm{s}}=r_{1}^{\mathrm{b}} r_{2}^{\mathrm{b}}
$$

By eliminating the monomer concentrations from the equations (1), (5) and (6) the following equation can be obtained:

$$
p_{12}^{2}\left(r_{1} r_{2}-1\right) F_{1}+p_{12}+F_{1}-1=0
$$

with $r_{1} \neq 0$. When $r_{1}=0: p_{12}=1$ and equation (13) becomes invalid; although, when $r_{2}=0$, the equation is still valid.
Solving this equation yields the following expression for $p_{12}$ :

$$
p_{12}=\frac{1-\left[4 F_{1}^{2}\left(1-r_{1} r_{2}\right)-4 F_{1}\left(1-r_{1} r_{2}\right)+1\right]^{1 / 2}}{2 F_{1}\left(1-r_{1} r_{2}\right)}
$$

with $r_{1} r_{2} \neq 1$.

It becomes clear that if the bootstrap model applies the triad distribution versus copolymer composition is independent of the solvent. This can be seen easily from the dependence of the triad distribution on $p_{12}$, which is only dependent on the product $r_{1} r_{2}$. This product was shown to be independent of solvent. It is interesting to note that the square root function in equation (14) equals the quantity $\kappa$ defined by Stockmayer when he mathematically derived the distribution of chain lengths and compositions in copolymers ${ }^{8}$.

For the penultimate unit model a similar derivation can be used and the following set of equations applies ${ }^{9}$ :

$$
\begin{aligned}
\frac{F_{1}}{F_{2}} & =\frac{\left(1+\frac{p_{211}}{p_{112}}\right)}{\left(1+\frac{p_{122}}{p_{221}}\right)} \\
F_{111} & =\frac{p_{211}\left(1-p_{112}\right)}{p_{112}+p_{211}} \\
F_{112}+F_{211} & =\frac{2 p_{112} p_{211}}{p_{112}+p_{211}} \\
F_{212} & =\frac{p_{112}\left(1-p_{211}\right)}{p_{112}+p_{211}}
\end{aligned}
$$

The conditional probabilities in this case can be written in a way similar to the Mayo-Lewis model, using the distribution coefficient $K$ :

$$
\begin{aligned}
& p_{112}=\frac{1}{1+r_{11} K q} \\
& p_{211}=\frac{r_{21} K q}{1+r_{21} K q} \\
& p_{221}=\frac{1}{1+\frac{r_{22}}{K q}} \\
& p_{122}=\frac{\frac{r_{12}}{K q}}{1+\frac{r_{12}}{K q}}
\end{aligned}
$$

where $K$ and $q$ are defined in the same way as in the derivation for the Mayo-Lewis model. The reactivity ratios $r_{i j}$ are defined as:

$$
r_{i j}=\frac{k_{i j j}}{k_{i j k}}
$$

where subscript $i, j$ and $k$ may be 1 or 2 and subscript $k \neq$ subscript $j$. From equations (19)-(22) it can be seen that the apparent reactivity ratios in a solvent are defined as

$$
\begin{aligned}
& r_{i 1}^{\mathrm{s}}=r_{i 1} K^{\mathrm{s}} \\
& r_{i 2}^{\mathrm{s}}=\frac{r_{i 2}}{K^{\mathrm{s}}}
\end{aligned}
$$


If we define the distribution coefficient $K^{\mathrm{b}}$ to be unity for bulk copolymerization, analogous to the derivation using the Mayo-Lewis model it follows in this case:

$$
\begin{aligned}
& \frac{r_{i 1}^{\mathrm{s}}}{r_{i 1}^{\mathrm{b}}}=K^{\mathrm{s}} \\
& \frac{r_{i 2}^{\mathrm{b}}}{r_{i 2}^{\mathrm{s}}}=K^{\mathrm{s}}
\end{aligned}
$$

The derivation of the equations describing the triad distribution as a function of polymer composition for the penultimate unit model appears to be complicated if no constraints are applied. However, for styrene maleic anhydride (STY/MAnh) copolymers the assumption is allowed that no homopropagation of monomer 2 (MAnh) occurs. Thus, equation (15) simplifies to

$$
\frac{F_{1}}{F_{2}}=1+\frac{p_{211}}{p_{112}}
$$

With this simplification $p_{112}$ and $p_{211}$ can be expressed as a function of copolymer composition

$$
\begin{aligned}
& p_{211}=\frac{\frac{F_{1}}{F_{1}-1}+\left[\left(\frac{F_{1}}{1-F_{1}}\right)^{2}+4\left(\frac{2 F_{1}-1}{1-F_{1}}\right)\left(\frac{r_{11}}{r_{21}}-1\right)\right]^{1 / 2}}{2\left(\frac{r_{11}}{r_{21}}-1\right)} \\
& p_{112}=\frac{-F_{1}+\left(1-F_{1}\right)\left[\left(\frac{F_{1}}{1-F_{1}}\right)^{2}+4\left(\frac{2 F_{1}-1}{1-F_{1}}\right)\left(\frac{r_{11}}{r_{21}}-1\right)\right]^{1 / 2}}{2\left(2 F_{1}-1\right)\left(\frac{r_{11}}{r_{21}}-1\right)}
\end{aligned}
$$

This implies that if no homopropagation of monomer 2 is present the triad distribution versus copolymer composition is only dependent on $r_{11} / r_{21}$. Knowing that the simple Mayo-Lewis model is a special case of the penultimate unit model, the dependence of the triad distribution versus copolymer composition on the product $r_{1} r_{2}$ (or in this case $r_{11} r_{22}$ ) should be present too. Due to the symmetry in the copolymerization models the third factor in determining the triad distribution versus copolymer composition has to be $r_{22} / r_{12}$. Numerical inspection of these conditions using a computer model of the penultimate unit model confirms the three values to be the necessary and sufficient conditions for the triad distribution versus copolymer composition to be fixed. With due regard to the bootstrap model as put forward in equations (24) and (25) it is obvious that $r_{11} r_{22}, r_{11} / r_{21}$ and $r_{22} / r_{12}$ are independent of the distribution coefficient $K$.

We presently are aware that the bootstrap model cannot only apply to the Mayo-Lewis model, but also to the penultimate unit model and presumably others. Once it is clear that a comonomer pair behaves according to either Mayo-Lewis or penultimate unit model it is possible to determine distribution coefficients in a reference state as outlined above. Equations (10) and (11) should be used to determine $K$ values for the Mayo-Lewis model and equations (26) and (27) for the penultimate unit model.
Table 1 Apparent reactivity ratios for STY/MMA as calculated with the EVM method from data by San Roman et al. ${ }^{3}$ and distribution coefficients $K$ calculated according to equations (10) and (11)

\begin{tabular}{lccccc}
\hline Solvent & $r_{\mathrm{M}}$ & $r_{\mathrm{S}}$ & $r_{\mathrm{M}} r_{\mathrm{S}}$ & $K$ (from $t_{\mathrm{M}}$ ) & $K$ (from $r_{\mathrm{s}}$ ) \\
\hline Benzene & $0.44^{a}$ & 0.51 & 0.23 & 0.97 & 0.92 \\
& $(0.43)^{b}$ & $(0.51)$ & $(0.22)$ & $(0.95)$ & $(0.93)$ \\
Chlorobenzene & 0.47 & 0.43 & 0.20 & 1.04 & 1.10 \\
& $(0.49)$ & $(0.47)$ & $(0.23)$ & $(1.09)$ & $(1.00)$ \\
Benzonitrile & 0.65 & 0.47 & 0.31 & 1.44 & 1.00 \\
& $(0.62)$ & $(0.38)$ & $(0.24)$ & $(1.36)$ & $(1.24)$ \\
Bulk $^{13}$ & 0.45 & 0.47 & 0.21 & 1.00 & 1.00 \\
\hline
\end{tabular}

${ }^{a}$ Polymer composition determined by n.m.r.

${ }^{b}$ Polymer composition determined by carbon analysis

\section{Re-analysis of data on STY/MMA}

STY/MMA is known to behave according to the Mayo-Lewis model with respect to copolymer composition and microstructure ${ }^{10,11}$. Data by San Roman et al. ${ }^{3}$ is re-analysed using the error in variables method ${ }^{12}$. The calculated values for the apparent reactivity ratios and their product is shown in Table 1.

The distribution coefficient can be calculated using equations (10) and (11) and the values for the reactivity ratios in bulk as reported by O'Driscoll et al. ${ }^{13}$. The distribution coefficient calculated from $r_{1}$ and $r_{2}$ are also given in Table 1.

The qualitative conclusion of Davis that the results of STY/MMA copolymerizations can be interpreted using the bootstrap model can now be quantified. It appears that the distribution coefficient $K$ increases when changing the solvent from benzene $<$ bulk $<$ chlorobenzene $<$ benzonitrile. The physical background of this phenomenon has been reported earlier ${ }^{14}$. Without involving the bootstrap model Russo correlated the variation in reactivity ratio to the polarity of the solvent. Now, taking into account the bootstrap model, one may conclude that the difference in polarity between solvent and site of polymerization (polymer coil) is the determining factor with respect to the distribution coefficient.

\section{STY/MAnh copolymerizations}

For the STY/MAnh comonomer pair three different copolymerization conditions were studied. There is a high tendency to form alternating copolymers and, therefore, composition drift can occur very readily. It is for this reason that copolymerizations at a higher temperature were carried out using continuous copolymerization in a stirred tank reactor. Steady state conditions were used to ensure a constant comonomer ratio and thus a uniform copolymer composition. A disadvantage of the continuous copolymerization route, at least for this comonomer pair, is that the monomer ratio in the reactor is difficult to determine. However, for experiments where copolymer microstructure is studied as a function of copolymer composition the method appears to be valuable. For the determination of the apparent reactivity ratios in the case of STY/MAnh precipitation polymerization in toluene, an approach similar to the one described by Tidwell and Mortimer ${ }^{15}$ was used. This work will be described elsewhere ${ }^{16}$.

\section{EXPERIMENTAL}

\section{Materials}

For the batch experiments styrene was distilled at reduced pressure under nitrogen, to remove the inhibitor. 
All solvents employed were dried using the usual techniques and stored over molecular sieves. All other chemicals were high purity grades and were used without further purification. For the batch experiments the required amounts of monomers, solvent and initiator were accurately weighed into the glass reaction vessels, thoroughly freeze-thaw degassed, and closed under vacuum. The polymerizations were carried out at $60^{\circ} \mathrm{C}$ to conversions less than $3 \mathrm{wt} \%$. As the polymerizations were carried out in toluene, the copolymers precipitated on formation. The polymers were purified twice by reprecipitation from THF solution into isopropanol. The purified polymers were dried in vacuo at $50^{\circ} \mathrm{C}$ for $16 \mathrm{~h}$.

For the continuous copolymerizations the required amounts of monomers, solvent and initiator were weighed into a storage vessel and kept under nitrogen. The mixture was pumped into a stainless steel reactor which was maintained at $110^{\circ} \mathrm{C}$. After three times the mean residence time a polymer sample was collected. The polymers were recovered from solution and purification was carried out twice by reprecipitation from THF solution into isopropanol. The purified copolymers were dried in vacuo at $50^{\circ} \mathrm{C}$ for $16 \mathrm{~h}$.

\section{Characterization}

DEPT ${ }^{13} \mathrm{C}$ n.m.r. spectra were recorded on a Varian XL300 spectrometer at ambient temperature. Solutions were approximately $10 \% \mathrm{w} / \mathrm{v}$ in acetone $\mathrm{D}_{6}$ for samples with high MAnh content or in $\mathrm{CDCl}_{3}$ for samples with lower MAnh content. The compositions of the copolymers were determined from the triad fractions determined by DEPT ${ }^{13} \mathrm{C}$ n.m.r. and using i.r. spectroscopy, from the ratio between the relative extinctions $E_{1855 \mathrm{~cm}^{-1}} /$ $E_{1490 \mathrm{~cm}^{-1}}$. The latter method was calibrated against non-aqueous titration. The DEPT ${ }^{13} \mathrm{C}$ n.m.r. technique to determine styrene centred triad distributions has been described earlier ${ }^{17}$.

\section{RESULTS}

The experimental results are summarized in Table 2. The styrene centred triad distribution versus copolymer composition is shown in Figure 1, together with data from Hill et $a .^{18}$, who studied the copolymerization of STY/MAnh at $60^{\circ} \mathrm{C}$ in bulk. The data all seem to fit on the same set of curves, thus indicating the occurrence of the bootstrap effect. From equations (9)-(11), (15) and (16) it appears that the copolymer triad distribution versus composition only depends on the quotient $r_{11} / r_{21}$. This means that as in the STY/MMA case, where only the product $r_{1} r_{2}$ could be determined, only this quotient can be determined. Carrying out a non-linear fitting procedure gives $r_{11} / r_{21}=0.63$. The triad distribution calculated from equations $(16)-(18),(29)$ and $(30)$ for this value is shown in Figure $l$ as drawn curves.

\section{DISCUSSION}

The copolymerization of STY/MAnh has received considerable attention in the literature. Various models have been used to try to describe this copolymerization. The complex participation model and the penultimate

Table 2 Copolymer composition and microstructure data. Free radical copolymerization of STY with MAnh at $110^{\circ} \mathrm{C}($ butanone $)$ and $60^{\circ} \mathrm{C}($ toluene $)$

\begin{tabular}{|c|c|c|c|c|c|c|}
\hline \multirow[b]{2}{*}{ Solvent } & \multirow{2}{*}{$\begin{array}{l}f(\text { MAnh }) \\
\text { feed }\end{array}$} & \multirow{2}{*}{$\begin{array}{l}\text { F(MAnh) } \\
\text { n.m.r. }\end{array}$} & \multirow{2}{*}{$\begin{array}{l}\text { Composition } \\
\text { i.r. }\end{array}$} & \multicolumn{3}{|c|}{ Styrene centred triad distribution } \\
\hline & & & & SSS & $\mathrm{MSS}+\mathrm{SSM}$ & MSM \\
\hline \multirow[t]{21}{*}{ Butanone } & - & 0.227 & 0.237 & 0.486 & 0.439 & 0.074 \\
\hline & - & 0.129 & 0.112 & 0.724 & 0.256 & 0.020 \\
\hline & - & 0.143 & 0.145 & 0.691 & 0.284 & 0.025 \\
\hline & - & 0.168 & 0.167 & 0.630 & 0.336 & 0.034 \\
\hline & - & 0.196 & 0.183 & 0.558 & 0.398 & 0.044 \\
\hline & - & 0.213 & 0.221 & 0.503 & 0.451 & 0.045 \\
\hline & - & 0.230 & 0.242 & 0.455 & 0.494 & 0.051 \\
\hline & - & 0.268 & 0.264 & 0.367 & 0.535 & 0.039 \\
\hline & - & 0.286 & 0.284 & 0.331 & 0.538 & 0.131 \\
\hline & - & 0.303 & 0.295 & 0.294 & 0.545 & 0.162 \\
\hline & - & 0.320 & 0.311 & 0.248 & 0.564 & 0.189 \\
\hline & - & 0.337 & 0.329 & 0.222 & 0.540 & 0.238 \\
\hline & - & 0.350 & 0.349 & 0.183 & 0.539 & 0.278 \\
\hline & - & 0.375 & 0.370 & 0.151 & 0.497 & 0.352 \\
\hline & - & 0.231 & 0.231 & 0.463 & 0.475 & 0.069 \\
\hline & - & 0.253 & 0.251 & 0.412 & 0.500 & 0.088 \\
\hline & - & 0.285 & 0.272 & 0.319 & 0.556 & 0.125 \\
\hline & - & 0.296 & 0.292 & 0.297 & 0.567 & 0.136 \\
\hline & - & 0.308 & 0.313 & 0.270 & 0.567 & 0.163 \\
\hline & - & 0.344 & 0.333 & 0.190 & 0.568 & 0.241 \\
\hline & - & 0.348 & 0.374 & 0.184 & 0.563 & 0.253 \\
\hline \multirow[t]{10}{*}{ Toluene } & 0.160 & 0.458 & - & 0.00 & 0.31 & 0.69 \\
\hline & 0.500 & 0.485 & - & 0.00 & 0.12 & 0.88 \\
\hline & 0.760 & 0.500 & - & 0.00 & 0.00 & 1.00 \\
\hline & 0.062 & - & 0.378 & - & - & - \\
\hline & 0.064 & - & 0.378 & - & - & - \\
\hline & 0.060 & - & 0.367 & 0.10 & 0.50 & 0.40 \\
\hline & 0.059 & - & 0.375 & - & - & - \\
\hline & 0.010 & - & 0.210 & - & - & - \\
\hline & 0.009 & - & 0.197 & 0.49 & 0.45 & 0.06 \\
\hline & 0.010 & - & 0.208 & - & - & - \\
\hline
\end{tabular}




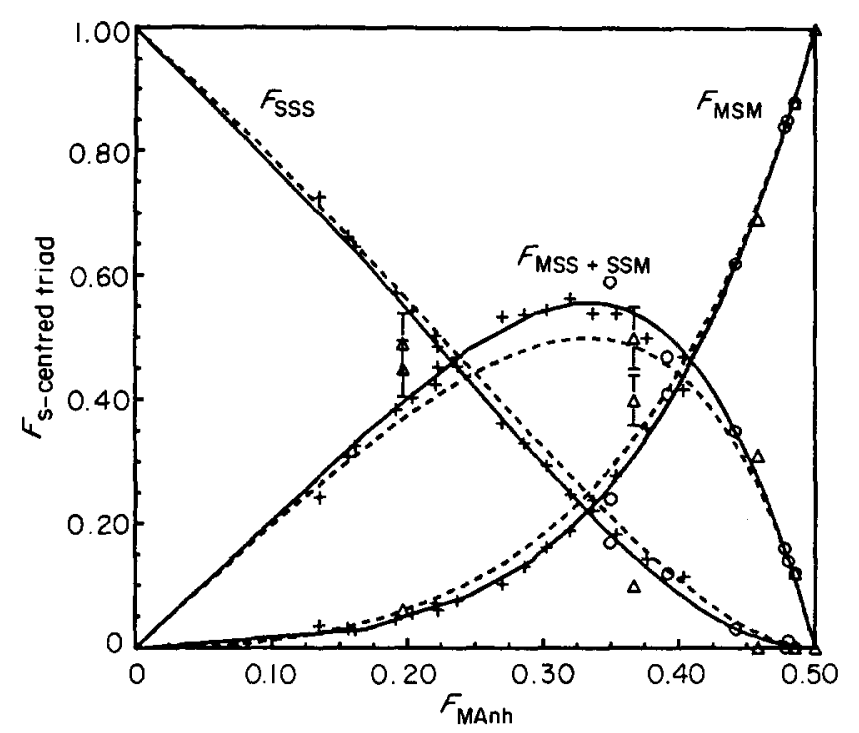

Figure 1 Mole fraction of STY centred triads versus mole fraction of MAnh in the STY/MAnh copolymer: $(+)$ butanone, $110^{\circ} \mathrm{C} ;(\mathrm{O})$ bulk, $60^{\circ} \mathrm{C} ; \triangle(\triangle)$ toluene, $60^{\circ} \mathrm{C}$. Dashed curves are the Mayo-Lewis model predictions with $r_{1} r_{2}=0$. Drawn curves are the penultimate unit model predictions with $r_{11} / r_{21}=0.63$

unit model appear to be the best in fitting the experimental data. In this work only the penultimate unit model is used. Since Harwood suggested ${ }^{1}$ that the bootstrap effect may cause or influence previously reported penultimate effects, the triad distribution versus copolymer composition data were also tested against the classical Mayo-Lewis model. However, if $r_{2}=0$ (no homopropagation of MAnh), then the product $r_{1} r_{2}=0$ and therefore the triad distribution versus copolymer composition is fixed. This model shows poor agreement with the experimental data (dashed curves in Figure 1). As shown, the experimental data can be described well using the penultimate unit model with $r_{11} / r_{21}=0.63$.

Apparent reactivity ratios were estimated from unpublished data on continuous polymerization in butanone at $110^{\circ} \mathrm{C}$. The calculated values were $r_{11}=0.20$ and $r_{21}=0.33$. Although these values, compared with values reported in literature ${ }^{19}$, show that reactivity ratios vary considerably with temperature, no effect is observed with respect to the ratio $r_{11} / r_{21}$. This may originate from activation energy of the various propagation rate constants and/or from the temperature dependence of the distribution coefficient. While the terminal monomer unit of the propagating chain and the incoming monomer are the same, the activation energy of $k_{11 i}$ and $k_{21 i}$ will be approximately the same. There may however exist a difference between $k_{j 12}$ and $k_{j 11}$. Therefore, it is possible that $r_{11}$ and $r_{21}$ differ, but that the quotient remains constant. On the other hand it is known that there is only a slight influence of temperature on apparent reactivity ratios. If, in the extreme case, reactivity ratios may be regarded as independent of temperature, values for the distribution coefficient may be calculated for butanone at $110^{\circ} \mathrm{C}$. It is found that $K=11.3$ can be calculated from $r_{11}$ and $K=11.5$ from $r_{21}$. Reference values for bulk copolymerization were taken from the work by Hill et al. ${ }^{18}\left(r_{11}=0.0177 ; r_{21}=0.0288\right)$.

From the copolymer composition versus monomer feed data on the polymerizations in toluene, apparent reactivity ratios were calculated using a non-linear least squares method. It was found that $r_{11}=0.021$ and $r_{21}=0.049$ or $r_{11} / r_{21}=0.43$. If reactivity ratios are also calculated from the five experiments where monomer feed ratio, copolymer composition and triad distribution are known the values are $r_{11}=0.022$ and $r_{21}=0.029$ or $r_{11} / r_{21}=0.76$. The data is scattered for polymerizations in toluene, shown by the error bars in Figure 1 for two of the samples. The polymer precipitates on formation in toluene. Therefore, the scatter may be an artifact caused by the heterogeneity of the system. An additional source of error may arise from the low MAnh content in the monomer feed of some of the experiments, which readily leads to composition drift. If, notwithstanding the uncertainty in the reactivity ratio values, $K$-values for toluene are calculated from the latter $r_{11}$ and $r_{21}$ (since these are based on the largest amount of information), the following values are estimated. Calculated from $r_{11}$ it is found that $K=1.2$ and calculated from $r_{21}$ it is found that $K=1.0$.

\section{CONCLUSIONS}

From the present work it can be concluded that quantification of the bootstrap effect is possible. It should be emphasized that the choice of bulk copolymerization as reference state does not necessarily mean that in this case global comonomer ratio equals monomer ratio at the site of propagation. The bootstrap effect can apply to the Mayo-Lewis model and the penultimate unit model. In either case the copolymer microstructure versus composition data does not provide information about the individual reactivity ratio values or about the distribution coefficient. Such data only provide information about certain reactivity ratio product or quotient values.

\section{ACKNOWLEDGEMENTS}

Support for K. F. O'Driscoll by the Natural Science and Engineering Research Council of Canada is appreciated. The authors wish to thank Mr Smids, Mr Maassen and Ms Vonk (DSM research) for the synthesis of the STY/MAnh copolymers and Dr Pionteck (University of Massachusetts) for providing the DEPT ${ }^{13} \mathrm{C}$ n.m.r. data. We are indebted to $\mathrm{Dr}$ G. Moad for pointing out the possibility of ambiguity concerning the nature of the data used by Davis.

\section{REFERENCES}

1 Harwood, H. J. Makromol. Chem., Macromol. Symp. 1987, 10/11, 331

2 San Roman, J., Madruga, E. L. and Del Puerto, M. A. Angew. Makromol. Chem. 1979, 78, 129

3 San Roman, J., Madruga, E. L. and Del Puerto, M. A. Angew. Makromol. Chem. 1980, 86, 1

4 San Roman, J. and Madruga, E. L. Angew. Makromol. Chem. 1980, 86, 17

5 Davis, T. P. Polymer Commun. 1990, 31, 442

6 San Roman, J. Personal communication, 1991

7 Bovey, F. A. J. Polym. Sci. 1962, 62, 197

8 Stockmayer, W. H. J. Chem. Phys. 1945, 13, 199

9 Hill, D. J. T. and O'Donnell, J. H. Makromol. Chem., Macromol. Symp. 1987, 10/11, 375

10 Fukuda, T., Ma, Y.-D. and Inagaki, H. Macromolecules 1985, 18, 17

11 O'Driscoll, K. F. and Davis, T. P. J. Polym. Sci., Polym. Lett. Edn. 1989, 27, 417 


\section{Bootstrap model in styrene copolymerization: B. Klumperman and K. F. O'Driscoll}

12 Dube, M., Sanayei, R. A., Penlidis, A., O'Driscoll, K. F. and Reilly, P. M. J. Polym. Sci., Polym. Chem. Edn. 1991, 29, 703

13 O'Driscoll, K. F., Kale, L. T., Garcia Rubio, L. H. and Reilly, P. M. J. Polym. Sci., Chem. Edn. 1984, 22, 2777

14 Russo, S. Polymer 1975, 16, 429

15 Tidwell, P. W. and Mortimer, G. A. J. Polym. Sci. A 1965, 3, 369
16 Klumperman, B. and Vonk, G. in preparation

17 Barron, P. F., Hill, D. J. T., O'Donnell, J. H. and O'Sullivan, P. W. Macromolecules 1984, 17, 1967

18 Hill, D. J. T., O'Donnell, J. H. and O'Sullivan, P. W. Macromolecules 1985, 18, 9

19 Chen, S. A. and Chang, G. Y. Makromol. Chem. 1986, 187, 1597 